\title{
Silicon-based spin quantum computation and the shallow donor exchange gate
}

\author{
Belita Koiller*, R. B. Capaz*, X. Hu ${ }^{\dagger}$ and S. Das Sarma** \\ *Instituto de Física, Universidade Federal do Rio de Janeiro, Cx. P. 68528, $21941-972$ Rio de Janeiro, Brazil \\ ${ }^{\dagger}$ Department of Physics, University at Buffalo, the State University of New York, Buffalo, NY 14260-1500 \\ ${ }^{* *}$ Condensed Matter Theory Center, Department of Physics, University of Maryland, MD 20742-4111
}

\begin{abstract}
Proposed silicon-based quantum-computer architectures have attracted attention because of their promise for scalability and their potential for synergetically utilizing the available resources associated with the existing Si technology infrastructure. Electronic and nuclear spins of shallow donors (e.g. phosphorus) in Si are ideally suited candidates for qubits in such proposals, where shallow donor exchange gates are frequently invoked to perform two-qubit operations. An important potential problem in this context is that intervalley interference originating from the degeneracy in the Si conduction-band edge causes fast oscillations in donor exchange coupling, which imposes significant constraints on the Si quantum-computer architecture. We discuss the theoretical origin of such oscillations. Considering two substitutional donors in $\mathrm{Si}$, we present a systematic statistical study of the correlation between relative position distributions and the resulting exchange distributions.
\end{abstract}

\section{INTRODUCTION}

As semiconductor devices decrease in size, their physical properties tend to become increasingly sensitive to the actual configuration of dopant substitutional impurities [1]. A striking example is the proposal of donor-based silicon quantum computer (QC) by Kane [2], in which the monovalent ${ }^{31} \mathrm{P}$ impurities in $\mathrm{Si}$ are the fundamental quantum bits (qubits). This intriguing proposal has created considerable recent interest in revisiting all aspects of the donor impurity problem in silicon, particularly in the $\mathrm{Si}:{ }^{31} \mathrm{P}$ system.

Two-qubit operations for the donor-based Si QC architecture, which are required for a universal $\mathrm{QC}$, involve precise control over electron-electron exchange[2, 3, 4] and electron-nucleus hyperfine interactions (for nuclear spin qubits). Such control can presumably be achieved by fabrication of donor arrays with accurate positioning and surface gates whose potential can be precisely controlled $[5,6,7,8]$. However, electron exchange in bulk silicon has spatial oscillations [9] on the atomic scale due to the valley interference arising from the particular sixfold degeneracy of the bulk Si conduction band. These oscillations place heavy burdens on device fabrication and coherent control [10], because of the very high accuracy requirement for placing each donor inside the $\mathrm{Si}$ unit cell, and/or for controlling the external gate voltages.

The potentially severe consequences of these problems for exchange-based Si QC architecture motivated us and other researchers to perform further theoretical studies, going beyond some of the simplifying approximations in the formalism adopted in Ref. [10], and incorporating perturbation effects due to applied strain[11] or gate fields [12]. These studies, performed within the standard Heitler-London (HL) formalism [13], essentially reconfirm the originally reported difficulties regarding the sensitivity of the electron exchange coupling to donor positioning, indicating that these may not be completely overcome by applying strain or electric fields. The sensitivity of the calculated exchange coupling to donor relative position originates from interference between the plane-wave parts of the six degenerate Bloch states associated with the Si conduction-band minima. More recently [14] we have assessed the robustness of the HL approximation for the two-electron donor-pair states by relaxing the phase pinning at donor sites, which could in principle eliminate the oscillatory exchange behavior. Within this more general theoretical scheme, the floating-phase HL approach, our main conclusion is that, for all practical purposes, the previously adopted HL wavefunctions are robust, and the oscillatory behavior obtained in Refs. [10,11,12] cannot be taken as an artifact.

\section{SINGLE DONOR}

We describe the single donor electron ground state using effective mass theory. The bound donor electron Hamil- 


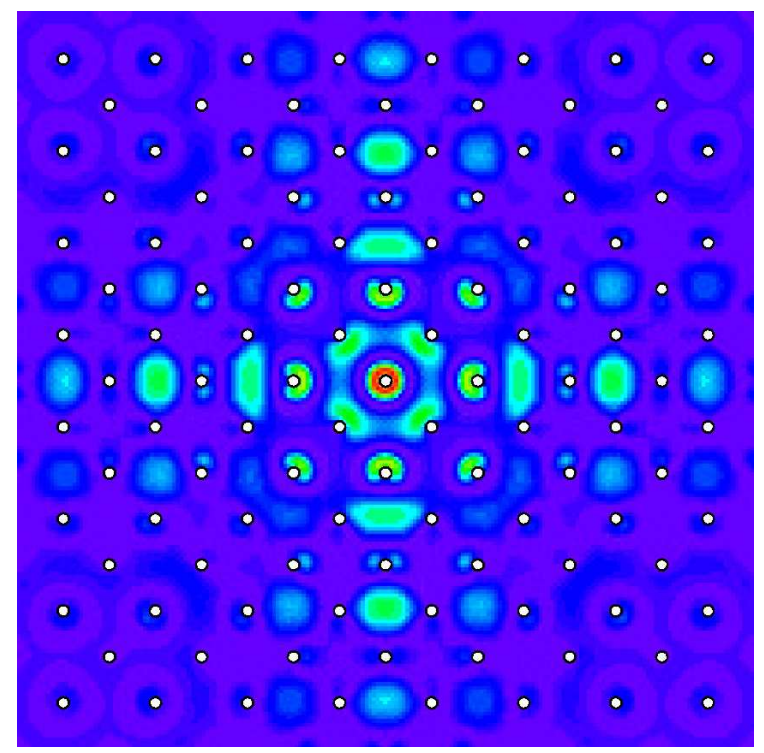

FIGURE 1. Electron probability density on the (001) plane of bulk Si for the ground state of a donor in $\mathrm{Si}$ within the envelope function approximation. The white dots give the inplane atomic sites. The color scheme runs from red (high density) to purple (low density). The highest density is at the central site, corresponding to the impurity site.

tonian for an impurity at site $\mathbf{R}_{0}$ is written as

$$
\mathscr{H}_{0}=\mathscr{H}_{S V}+\mathscr{H}_{V O}
$$

The first term, $\mathscr{H}_{S V}$, is the single-valley Kohn-Luttinger Hamiltonian [15], which includes the single particle kinetic energy, the Si periodic potential, and the impurity screened Coulomb perturbation potential

$$
V(\mathbf{r})=-\frac{e^{2}}{\varepsilon\left|\mathbf{r}-\mathbf{R}_{0}\right|} .
$$

For shallow donors in $\mathrm{Si}$, we use the static dielectric constant $\varepsilon=12.1$. The second term of Eq. (1), $\mathscr{H}_{V O}$, represents the inter-valley scattering effects due to the presence of the impurity which breaks the bulk translational symmetry.

The electron eigenfunctions are written on the basis of the six unperturbed Si band edge Bloch states $\phi_{\mu}=$ $u_{\mu}(\mathbf{r}) \mathbf{e}^{\mathbf{i} \mathbf{k}_{\mu} \cdot \mathbf{r}}$ [recall that the conduction band of bulk Si has six degenerate minima $(\mu=1, \ldots, 6)$, located along the $\Gamma-\mathrm{X}$ axes of the Brillouin zone at $\left|\mathbf{k}_{\mu}\right| \sim 0.85(2 \pi / \mathrm{a})$ from the $\Gamma$ point]:

$$
\psi_{\mathbf{R}_{0}}(\mathbf{r})=\frac{1}{\sqrt{6}} \sum_{\mu=1}^{6} F_{\mu}\left(\mathbf{r}-\mathbf{R}_{0}\right) u_{\mu}(\mathbf{r}) e^{i \mathbf{k}_{\mu} \cdot\left(\mathbf{r}-\mathbf{R}_{0}\right)}
$$

The phases of the plane-wave part of all band edge Bloch states are naturally chosen to be pinned at $\mathbf{R}_{0}$ : In this way the charge density at the donor site [where the donor perturbation potential Eq. (2) is more attractive] is maximum, thus minimizing the energy for $\psi_{\mathbf{R}_{0}}(\mathbf{r})$.

In Eq. (3), $F_{\mu}\left(\mathbf{r}-\mathbf{R}_{0}\right)$ are envelope functions centered at $\mathbf{R}_{0}$, for which we adopt the anisotropic Kohn-Luttinger form, e.g., for $\mu=z, F_{z}(\mathbf{r})=\exp \left\{-\left[\left(x^{2}+y^{2}\right) / a^{2}+\right.\right.$ $\left.\left.z^{2} / b^{2}\right]^{1 / 2}\right\} / \sqrt{\pi a^{2} b}$. The effective Bohr radii $a$ and $b$ are variational parameters chosen to minimize $E_{S V}=$ $\left\langle\psi_{\mathbf{R}_{0}}\left|\mathscr{H}_{S V}\right| \psi_{\mathbf{R}_{0}}\right\rangle$, leading to $a=25 \AA, b=14 \AA$ and $E_{S V} \sim-30 \mathrm{meV}$ when recently measured effective mass values are used in the minimization [10]. The periodic part of each Bloch function is pinned to the lattice, independent of the donor site.

The $\mathscr{H}_{S V}$ ground state is six-fold degenerate. This degeneracy is lifted by the valley-orbit interactions [16], which are included here in $\mathscr{H}_{V O}$, leading to the nondegenerate ( $A_{1}$-symmetry) ground state in (3). Fig. 1 gives the charge density $\left|\psi_{\mathbf{R}_{0}}(\mathbf{r})\right|^{2}$ for this state, where the periodic part of the conduction band edge Bloch functions were obtained from $a b$-initio calculations, as described in Ref. [14]. The impurity site $\mathbf{R}_{0}$, corresponding to the higher charge density, is at the center of the frame. It is interesting that, except for this central site, regions of high charge concentration and atomic sites do not necessarily coincide, because the charge distribution periodicity imposed by the plane-wave part of the Bloch functions is $2 \pi / k_{\mu}$, incommensurate with the lattice period.

The oscillatory behavior of the single donor wave functions in Si, illustrated in Fig. 1, is well established experimentally [17] and theoretically $[14,18]$. This behavior does not bring significant consequences for conventional applications in Si-based devices (n-doped $\mathrm{Si}$ ). A recent study of the single-qubit operations ( $A$-gate) in the Kane QC shows that the $A$-gate operations do not present additional complications due to the Si band structure interference effects [19].

\section{DONOR PAIR EXCHANGE COUPLING}

The HL approximation is a reliable scheme for the wellseparated donor pair problem (interdonor distance much larger than the donor Bohr radii) [13]. Within HL, the lowest energy singlet and triplet wavefunctions for two electrons bound to a donor pair at sites $\mathbf{R}_{A}$ and $\mathbf{R}_{B}$, are written as properly symmetrized combinations of $\psi_{\mathbf{R}_{A}}$ and $\psi_{\mathbf{R}_{B}}$ [as defined in Eq.(3)]

$$
\begin{aligned}
\Psi_{t}^{s}\left(\mathbf{r}_{1}, \mathbf{r}_{2}\right) & =\frac{1}{\sqrt{2\left(1 \pm S^{2}\right)}}\left[\psi_{\mathbf{R}_{A}}\left(\mathbf{r}_{1}\right) \psi_{\mathbf{R}_{B}}\left(\mathbf{r}_{2}\right)\right. \\
& \left. \pm \psi_{\mathbf{R}_{B}}\left(\mathbf{r}_{1}\right) \psi_{\mathbf{R}_{A}}\left(\mathbf{r}_{2}\right)\right]
\end{aligned}
$$

where $S$ is the overlap integral and the upper (lower) sign corresponds to the singlet (triplet) state. The energy expectation values for these states, $E_{t}^{s}=\left\langle\Psi_{t}^{s}|\mathscr{H}| \Psi_{t}^{s}\right\rangle$, 


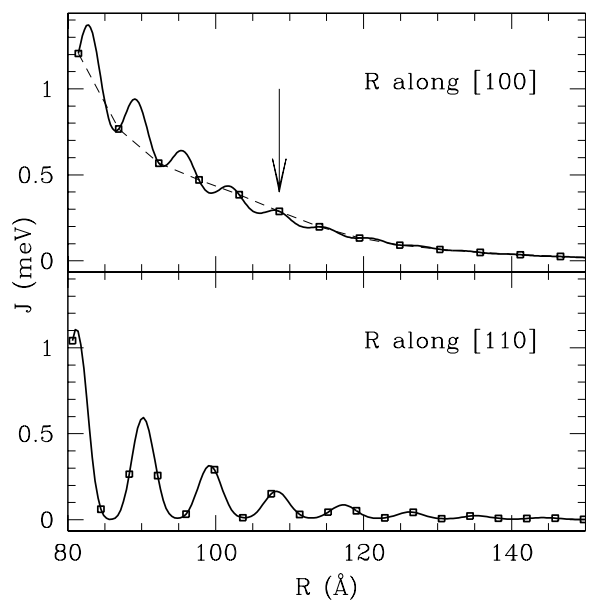

FIGURE 2. Exchange coupling between two phosphorus donors in $\mathrm{Si}$ along the indicated directions in the diamond structure. Values appropriate for impurities at substitutional sites are given by the squares. The dashed line in the $R \|[100]$ frame is a guide to the eye, indicating that the oscillatory behavior may be ignored for donors positioned exactly along this axis.

gives the exchange splitting through their difference, $J=E_{t}-E_{s}$. We have previously derived the expression for the donor electron exchange splitting $[11,14]$, which we reproduce here:

$$
J(\mathbf{R})=\frac{1}{36} \sum_{\mu, v} \mathscr{J}_{\mu v}(\mathbf{R}) \cos \left(\mathbf{k}_{\mu}-\mathbf{k}_{v}\right) \cdot \mathbf{R}
$$

where $\mathbf{R}=\mathbf{R}_{A}-\mathbf{R}_{B}$ is the interdonor position vector and $\mathscr{J}_{\mu \nu}(\mathbf{R})$ are kernels determined by the envelopes and are slowly varying $[10,11]$. Note that equation (5) does not involve any oscillatory contribution from $u_{\mu}(\mathbf{r})$, the periodic part of the Bloch functions [12, 14]. The physical reason for that is clear from (3): While the plane-wave phases of the Bloch functions are pinned to the donor sites, leading to the cossine factors in (5), the periodic functions $u_{\mu}$ are pinned to the lattice, regardless of the donor location.

The exchange energy calculated from Eq. (5) for a pair of donors as a function of their relative position along the [100] and [110] crystal axis is given in Fig. 2. This figure vividly illustrates both the anisotropic and the oscillatory behavior of $J(\mathbf{R})$, which is well established from previous studies $[9,10,11,12]$. It is interesting to note that for substitutional donors with interdonor position vectors exactly aligned with the [100] crystal axis, the oscillatory behavior may be ignored in practice, as indicated by the dashed line in the figure. This behavior is qualitatively similar to the exchange versus donor separation dependence assumed in Kane's proposal [2], where the Herring and Flicker expression [20], originally derived for $\mathrm{H}$ atoms, was adapted for donors in $\mathrm{Si}$. Therefore one might expect that reliable exchange gates operation would be possible if donors are exactly aligned along the [100] crystal axis.

\section{NANOFABRICATION ASPECTS}

Aiming at the fabrication of a P donor array accurately positioned along the [100] axis, and given the current degree of control in substitutional $\mathrm{P}$ positioning in $\mathrm{Si}$ of a few $\mathrm{nm}[5,6,7,8]$, we investigate the consequences of interdonor positioning uncertainties in the values of the corresponding pairwise exchange coupling. We define the target interdonor position $\mathbf{R}_{t}$ along [100], with an arbitrarily chosen length of 20 lattice parameters $(\sim 108.6$ $\AA$ ) indicated by the arrow in Fig. 2. The distributions for the interdonor distances $R=\left|\mathbf{R}_{A}-\mathbf{R}_{B}\right|$ when $\mathbf{R}_{A}$ is fixed and $\mathbf{R}_{B}$ "visits" all of the diamond lattice sites within a sphere centered at the target position are given in Fig. 3 . Different frames give results for different uncertainty radii, and, as expected, increasing the uncertainty radius results in a broader distribution around the target distance. Note that the geometry of the lattice implies that the distribution is always centered and peaked around $R_{t}$, as indicated by the arrows. The additional peaks in the distribution reveal the discrete nature of the Si lattice.

The respective distributions of exchange coupling between the same donor pairs in each ensemble is presented in Fig. 4, where the arrows give the exchange value at the target relative position: $J\left(\mathbf{R}_{t}\right) \sim 0.29 \mathrm{meV}$. The results here are qualitatively different from the distance distributions in Fig. 3, since they are neither centered nor peaked at the target exchange value. Even for the smallest uncertainty radius of $1 \mathrm{~nm}$ in (a), the exchange distribution is peaked around $J \sim 0$, bearing no semblance to the interdonor distance distributions. Increasing the uncertainty radius leads to a wider range of exchange values, with a more pronounced peak around the lowest $J$ values.

From the perspective of current QC fabrication efforts, $\sim 1 \mathrm{~nm}$ accuracy in single $\mathrm{P}$ atom positioning has been recently demonstrated [6], representing a major step towards the goal of obtaining a regular donor array embedded in single crystal Si. Distances and exchange coupling distributions consistent with such accuracy are presented in Figs.3(a) and 4(a) respectively. The present calculations indicate that such deviations in the relative position of donor pairs with respect to perfectly aligned substitutional sites along [100] lead to order-of-magnitude changes in the exchange coupling, favoring $J \sim 0$ values. Severe limitations in controlling $J$ would come from "hops" into different substitutional lattice sites. There- 


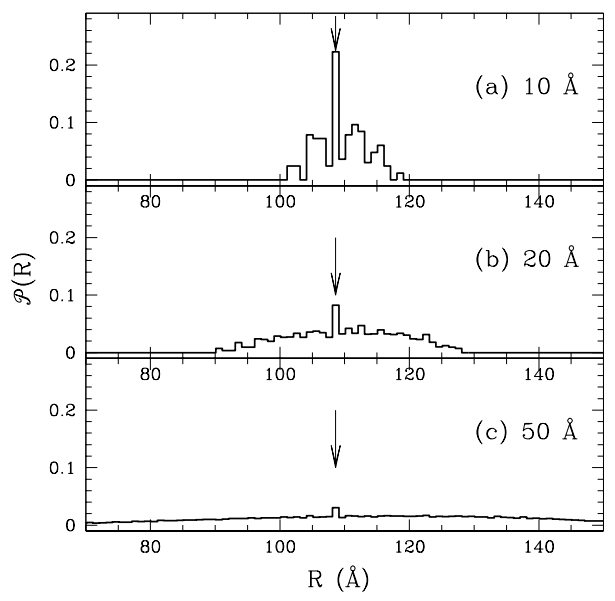

FIGURE 3. Interdonor distance distributions for a target relative position of 20 lattice parameters along [100] (see arrows). The first donor is fixed and the second one "visits" all of the $\mathrm{Si}$ substitutional lattice sites within a sphere centered at the target position, with uncertainty radii (a) $10 \AA$, (b) $20 \AA$ and (c) $50 \AA$.

fore, precisely controlling exchange gates in Si remains an open challenge.

\section{ACKNOWLEDGMENTS}

This work was partially supported by $\mathrm{CNPq}$ and by Instituto do Milênio de Nanociências in Brazil, by ARDA and LPS at the University of Maryland and by ARDA and ARO at the University at Buffalo.

\section{REFERENCES}

1. Voyles, P., Muller, D., Grazui, J., Citrin, P., and Grossmann, H.-J., Nature, 416, 827 (2002).

2. Kane, B., Nature, 393, 133 (1998).

3. Vrijen, R., Yablonovitch, E., Wang, K., Jiang, H., Balandin, A., Roychowdhury, V., Mor, T., and DiVincenzo, D., Phys. Rev. A, 62, 012306 (2000).

4. Hu, X., and Das Sarma, S., Phys. Rev. A, 61, 062301 (2000).

5. O'Brien, J. L., Schofield, S. R., Simmons, M. Y., Clark, R. G., Dzurak, A. S., Curson, N. J., Kane, B. E., McAlpine, N. S., Hawley, M. E., and Brown, G. W., Phys. Rev. B, 64, 161401 (2001).

6. Schofield, S. R., Curson, N. J., Simmons, M. Y., Rueß, F. J., Hallam, T., Oberbeck, L., and Clark, R. G., Phys. Rev. Lett., 91, 136104 (2003).

7. Buehler, T. M., McKinnon, R. P., Lumpkin, N. E., Brenner, R., Reilly, D. J., Macks, L. D., Hamilton, A. R.,

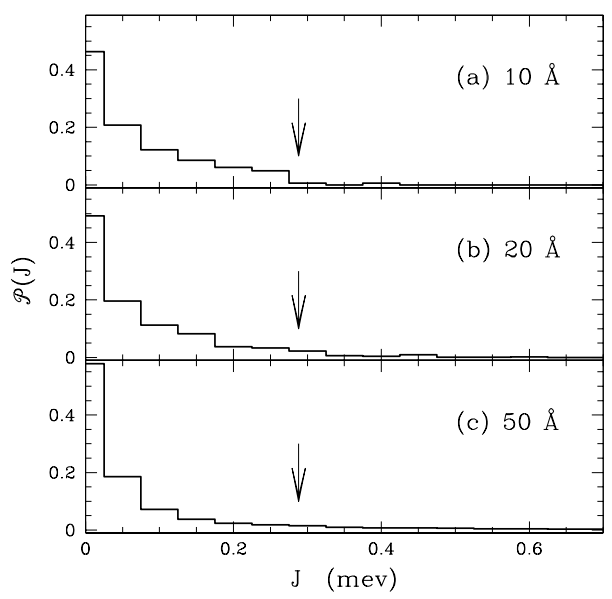

FIGURE 4. Exchange distributions for the same relative position ensembles in Fig. 3. The arrow indicates the target situation. Contrary to the distance distributions, the exchange distributions are not centered or peaked around the target value.

Dzurak, A. S., and Clark, R. G., Nanotechnology, 13, 686 (2002).

8. Schenkel, T., Persaud, A., Park, S. J., Nilsson, J., Bokor, J., Liddle, J. A., Keller, R., Schneider, D. H., Cheng, D. W., and Humphries, D. E., J. Appl. Phys., 94, 7017 (2003).

9. Andres, K., Bhatt, R. N., Goalwin, P., Rice, T., and Walstedt, R., Phys. Rev. B, 24, 244 (1981).

10. Koiller, B., Hu, X., and Das Sarma, S., Phys. Rev. Lett,, 88, 027903 (2002).

11. Koiller, B., Hu, X., and Das Sarma, S., Phys. Rev. B, 66, 115201 (2002).

12. Wellard, C. J., Hollenberg, L. C. L., Parisoli, F., Kettle, L., Goan, H.-S., McIntosh, J. A. L., and Jamieson, D. N., Phys. Rev. B, 68, 195209 (2003).

13. Slater, J. C., Quantum Theory of Molecules and Solids, vol. 1, McGraw-Hill, New York, 1963.

14. Koiller, B., Capaz, R. B., Hu, X., and Das Sarma, S., airXiv:cond-mat/0402266 (2004).

15. Kohn, W., Solid State Physics Series, vol. 5, Academic Press, 1957, edited by F. Seitz and D. Turnbull, p.257, and references therein.

16. Pantelides, S. T., Rev. Mod. Phys., 50 (1978).

17. Feher, G., Phys. Rev. B, 114, 1219 (1959).

18. Overhof, H., and Gerstmann, U., Phys. Rev. Lett., 92, 087602 (2004)

19. Martins, A. S., Capaz, R. B., and Koiller, B., Phys. Rev. B, 69, 085320 (2004).

20. Herring, C., and Flicker, M., Phys. Rev., 134, A362 (1964). 